\title{
On the prediction of core level binding energies in molecules, surfaces and solids
}

\author{
Francesc Viñes, Carmen Sousa, Francesc Illas* \\ Departament de Ciència de Materials i Química Física \& Institut de Quimica Teòrica i \\ Computacional (IQTCUB), Universitat de Barcelona, c/Martí i Franquès 1,08028 \\ Barcelona, Catalunya, Spain.
}

\begin{abstract}
Core level binding energies, directly measured with X-ray photoelectron spectroscopy (XPS), provide unique information regarding the chemical environment of atoms in a given system. However, interpretation of XPS in extended systems may not be straightforward and requires assistance from theory. The different state-of-the-art theoretical methods commonly used to approach core level binding energies and their shifts with respect to a given reference are reviewed and critically assessed with special emphasis on recent developed theoretical methods and with a focus on future applications in materials and surface sciences.
\end{abstract}

Keywords: X-Ray Photoemission Spectroscopy $・$ Core Levels $・$ Binding Energies $・$ Density Functional Calculations • Delta Self-Consistent Field

*corresponding author: francesc.illas@ub.edu 


\section{Introduction}

The Binding Energy (BE) of core electrons is characteristic of each chemical element and its accurate measurement was made possible thanks to the pioneering work of Siegbahn et al. back in $1957,{ }^{1}$ an outstanding achievement recognized by the 1981 Nobel Prize in Physics. The practical implementation of this new experimental technique provided the basis of the Electron Spectroscopy for Chemical Analysis (ESCA), ${ }^{2,3}$ a method of analysis which is routinely used at research centres in academy and industry. The experimental technique measures the kinetic energy $\left(E_{k i n}\right)$ of electrons produced when a system is irradiated by X-rays of known frequency $v$. The core level binding energy is directly provided by invoking Einstein's equation of the photoelectric effect

$$
\mathrm{h} v=\mathrm{BE}+\mathrm{E}_{\text {kin }}
$$

and can be applied to molecules in the gas phase in a straightforward way, see Scheme 1 of the prototypical case of $\mathrm{H}_{2} \mathrm{O}$ molecule. In the case of surfaces and solids it is necessary to consider an extra term; this is the surface work function $(\phi)$, accounting for the energy required to remove a free electron (at the Fermi level, $\mathrm{E}_{\mathrm{F}}$ ) from the solid and bring it to the vacuum, see a surface example in Scheme 1. Then, the BE is provide by

$$
\mathrm{h} v=\mathrm{BE}+\mathrm{E}_{\mathrm{kin}}+\phi
$$

Because of the accurate measurement of the surface work function is not simple, the application of this technique to materials science usually focus on the difference of BE for a given atom in different chemical environments. The quantity of interest is now $\Delta \mathrm{BE}$, usually taken with respect to a given reference; for instance, the BE of the element in the bulk most stable form. In the context of materials science, this technique is usually referred to as X-Ray Photoelectron Spectroscopy (XPS) and it is widely used since $\triangle \mathrm{BE}$ values provide information about the local environment of the element from which the photoelectron is removed, this involves for instance oxidation states ${ }^{4}$ and other bonding features which can 
only be disclosed by theoretical approaches. ${ }^{5,6}$ Moreover, the fact that the technique is surface sensitive ${ }^{2,} 3$ makes it especially suitable to investigate surface phenomena. Recently, XPS has been used to observe in situ the evolution of heterogeneously catalyzed reactions providing invaluable atomic level information about reaction mechanisms. ${ }^{7-9}$

The broad use of XPS in materials and surface sciences together to the applications in heterogeneous catalysis has triggered also a number of theoretical approaches aimed at predicting BEs or, most often, $\triangle \mathrm{BEs}$. The prediction of absolute BEs is straightforward for finite systems such as atoms, molecules, clusters or nanoparticles, provided a reliable method exists to approach the total energy of the neutral and core-ionized systems as described in detail in the next section. Starting from the seminal work of Bagus ${ }^{10}$ on the calculation of BEs at the Hartree-Fock level of theory, recent advances in the first-principles based methods of electronic structure will be emphasized. In these systems, comparison between theory and available experimental data (see for instance Ref. 11) can be carried out in a clear-cut way. A similar strategy is also possible for surfaces and solids as long as they can be described through an appropriate finite representation as in the cluster model approach. ${ }^{5}$

In the case of surfaces or solids described through periodic models, a common choice in computational materials science, the situation is less clear since the unit cell corresponding to the core-ionized system is charged and some action is needed to avoid artefacts arising from the coulombic repulsion between the periodically repeated infinite unit cells; these will be briefly described at the end of Section 4. Also, it is important to point out that in the case of extended systems the experimental measurements are often relative to a given arbitrary reference. For instance, it is customary to use the $\mathrm{C}(1 s)$ core level as reference and to assign to it a $\mathrm{BE}$ of $285 \mathrm{eV} .{ }^{12}$ In these situations the quantity of interest is $\triangle \mathrm{BE}$ rather than $\mathrm{BE}$ and this is the reason why most attention has been devoted to the calculation of the former values. ${ }^{5,13,14}$ Nevertheless, it is important to stress that interpretation of XPS is not always 
easy. The case of atomic $\mathrm{O}$ on $\mathrm{Al}(111)$ is a paradigmatic example where naïve interpretations of two well defined features is in contradiction with theoretical results leading in turn to an fully consistent alternative interpretation. ${ }^{15}$ In Section 2 of this perspective the available theoretical methods are described and critically assessed. Section 3 provides an example which can be used as a practicum, whereas Section 4 reports an overview of relevant results in the literature.

\section{Theoretical approaches to compute core level binding energies}

The $\mathrm{BE}$ of a given (core or valence) electronic level corresponds to the energy difference between the initial $\mathrm{N}$-electron non-ionized state and the $\mathrm{N}-1$ electron final ionized state:

$$
\mathrm{BE}=E^{\mathrm{N}-1}(\text { final })-E^{\mathrm{N}}(\text { initial })
$$

$\mathrm{BE}$ being positive for a bound state. In principle, the exact solution of the energy for the many-electron initial and final states could be obtained by full Configuration Interaction (CI) calculations within a relativistic Hamiltonian and an infinitely complete basis set. Obviously, this is not practicable and approximated methods are needed to estimate the total energy of a given system. Even within limited basis sets and/or truncated excitations, CI calculations are extremely complex since the proper solution for core level BEs corresponds to a highly excited root and convergence problems will certainly appear.

Two different situations can be encountered when computing BEs depending on whether the electronic states of interest are well described by a single configuration wave function or require a linear combination involving several configurations. ${ }^{13}$ In the first case one-body effects dominate and the measured spectra show a single main peak for each ionized electronic shell with weak satellites only. On the contrary, in the second case, many-body

effects are important and the spectra usually show several intense peaks for a given shell. Here we focus mainly on the first kind of systems, which is the most common situation in 
molecules containing main group elements. To describe the central features of the XPS spectra for these systems, monoconfigurational quantum chemistry methods can be used. In fact, self-consistent field (SCF) Hartree-Fock (HF) calculations have been extensively applied in the computation of core level BEs as a tool to understand and assign the peaks in XPS spectra. $^{5,10,13}$

At the HF level of theory, core level, as well as valence BEs can be approached in two ways. From Koopmans' theorem (KT) it follows that the negative value of the orbital energy $\left(-\varepsilon_{\mathrm{i}}\right)$ from which the electron is removed provides an estimate of the BE. It can be mathematically proven that the KT BE corresponds to the difference between the HF energy of the neutral molecule and the energy of the ionized system computed using the orbitals of the neutral molecule; the latter is often referred to as a Frozen Orbital (FO) approach. Clearly, KT predicted BEs are significantly larger than the experimental values since (final state) electron density relaxation effects in response to the presence of the electron hole are neglected. As a matter of fact, BEs computed based on KT give an estimation of the so-called initial state effects. These are mainly related to the chemical bonding and arise from the core and valence electron density of the atom before ionization. More accurate absolute values of BEs can be computed by taking the energy difference between the final ionized system and the initial neutral ground state as stated in Eq. 3, both obtained at the same HF level. The resulting approach is usually referred to as $\triangle \mathrm{SCF}$. This approach accounts for relaxation effects caused by the response of the passive electrons to the presence of the core hole and thus includes the so-called final state effects. Within the HF formalism, the relaxation energy, $E_{\mathrm{R}}$, is unambiguously defined as the difference between the KT and $\triangle \mathrm{SCF}$ BEs and, consequently, is always a positive value. For gas phase molecules containing light atoms, the errors of HF $\triangle \mathrm{SCF}$ computed BEs are typically of the order of $0.5-1.0 \mathrm{eV}$, the difference with respect to experiment due to the neglect of electron correlation and relativistic effects. ${ }^{16,17}$ As 
mentioned in the introduction, $\mathrm{BE}$ shifts $(\triangle \mathrm{BE})$ for a given atom in different chemical environments are often considered since absolute values of BEs are available for gas phase molecules only. A final comment is needed concerning spin symmetry since the neutral molecule exhibits most often a closed shell singlet ground state and, consequently, the core ionized molecule will exhibit a doublet ground state. Unless specified, all calculations described in the present work are spin restricted. Spin polarization effects can be taken into account by means of spin unrestricted calculations, these are most often negligible and indeed introduce uncontrolled spin contamination effects. Hence, the common practice is to rely on spin restricted calculations. ${ }^{13}$ Note, however, that in most solid state based codes, spin polarization is introduced with respect to an artificial closed shell reference state with half occupation in alpha and beta orbitals. In that case the effect can be as large as $10 \mathrm{eV}^{18}$ but we would argue that it is not appropriate to define it as spin polarization.

Core level BEs can also be approached by Density Functional Theory (DFT) based methods. For molecular systems, choosing the appropriate density functional to compute the total energies and determining BEs by applying Eq. 3 leads to calculated BEs comparable, or slightly better, to those obtained from the HF method. ${ }^{19-21}$ Note that DFT methods implicitly include exchange and correlation effects, even if to an unknown extent. However, important differences exist in the definition of initial and final state effects at the DFT level. It has been shown that Kohn-Sham orbital energies $\left(\mathrm{KS}-\varepsilon_{\mathrm{i}}\right)$ cannot be interpreted as a measure of absolute initial state contributions to the BEs, although they display correct $\triangle \mathrm{BE}$ shifts with respect to a given reference. ${ }^{16}$ In fact, there is an alternative, more physical, view to interpret KS orbitals as an approximation to Dyson orbitals. ${ }^{22,23}$ This point of view has been described in detail in the review papers by Ortiz ${ }^{24}$ and by Ortiz and Öhrn. ${ }^{25}$ Initial state effects in DFT calculations can be obtained by invoking the same physics, which means making use of a Frozen Orbital 
(FO) approach, where no response to the core hole is allowed. ${ }^{17}$ Hence, the FO density, $\rho(\mathrm{FO})$, is defined as:

$$
\rho_{\mathrm{i}}(\mathrm{FO})=\rho(\mathrm{gs})-\varphi_{\mathrm{i}}^{*} \varphi_{\mathrm{i}}
$$

which corresponds to the ground state density, $\rho(g s)$, but with a core-hole in the proper core orbital $\varphi_{\mathrm{i}}$. In this way, initial effects $\mathrm{BE}$ values can be obtained from:

$$
\mathrm{BE}_{\mathrm{i}}(\mathrm{FO})=E_{\mathrm{DFT}}\left[\rho_{\mathrm{i}}(\mathrm{FO})\right]-E_{\mathrm{DFT}}[\rho(\mathrm{gs})]
$$

where $E_{\mathrm{DFT}}$ denotes the energy computed with a particular density functional for both the ground state density and the density corresponding to the system with the core hole but with the density of the neutral molecule. This approach to quantify initial state effects in DFT computed BEs is equivalent to the use of $\mathrm{KT}$ in HF calculations. Accordingly, the relaxation energy, $E_{\mathrm{R}}$, is defined as the difference between the FO and DFT calculated BEs both obtained by applying Eq. 3 .

A potential approach beyond DFT based calculations to estimate ionization energies is the so-called GW quasiparticle approach introduced long ago by $\mathrm{Hedin}^{26}$ and reviewed later in a rigorous and pedagogical way by Aryasetiawan and Gunnarsson. ${ }^{27}$ This method is based on a generalization of the HF equations in terms of Green's functions (G), where the self-energy term is non-local and energy dependent and the electrostatic potential is dynamically screened (W) thus including explicitly many body effects. This methodology has been implemented at different levels of approximation, depending on whether the method is applied non-selfconsistently or self-consistently ${ }^{28}$ and, in any case, involves a power expansion of $\mathrm{W}$ usually truncated at the second order. A major drawback encountered by the approximated implementations of this approach is the possible dependence of the results on the starting electron density. This is the case when the method is not applied self-consistently although reliable results for the lowest ionization potential of 100 test molecules (the GW100 database $)^{29}$ have been reported at the simplest $\mathrm{G}_{0} \mathrm{~W}_{0}$ level where both $\mathrm{G}$ and $\mathrm{W}$ are computed 
from the electron density obtained from a standard DFT calculation within the generalized gradient approximation (GGA) type functionals. On the other hand, the computational resources needed increase drastically for quasiparticle self-consistent-GW calculations, although the results obtained applying this approach exhibit better accuracy. The GW method has been successfully applied in the field of materials and surface sciences in order to compute band gaps and electronic excitations. Concerning the calculation of BEs, the advantage of this method lies in the fact that quasiparticle energies of occupied levels effectively represent ionization potentials while for unoccupied states they represent electron affinities. Nevertheless, applications of this methodology to the prediction of core level BEs is still in its infancy with just one study about to be published. ${ }^{30}$ For a representative series of molecules, this study shows that self consistent GW quasiparticles provide a reliable estimate of core level BEs although the accuracy is still inferior to $\triangle \mathrm{SCF}$ with either HF or the various available, broadly used, density functionals, as exemplified in a forthcoming section.

Finally, one important issue that has to be considered in the computation of BEs is the influence of the relativistic effects as discussed in several reviews ${ }^{13}$ and highlighted since the days of the early $\triangle \mathrm{SCF}$ calculations. ${ }^{31}$ The importance of relativistic effects depends on the particular core under study, whether the ionized atom is heavy or light and the core hole involves $s$ shells or shells with non-zero orbital angular momentum. These effects can be quantified by explicit calculations of the relativistic effects, both scalar and spin-orbit contributions, on the molecular system, or by considering the relativistic correction on the $\mathrm{BE}$ determined in fully relativistic calculations of the isolated atoms. The latter approach has been shown to be reliable for molecular systems, like the CO molecule. ${ }^{32}$

\section{The $\mathrm{H}_{2} \mathrm{O}$ molecule $\mathrm{O}(1 s)$ core level as a textbook example}

As an illustrative example of the accuracy of the different computational approaches to compute BEs described above, we have studied the $\mathrm{O}(1 s)$ core hole in the water molecule. 
Calculations have been performed at the optimized geometry as obtained using the $\mathrm{PBE}^{33}$ functional and a tight Tier 2 numerical atom-centred orbitals basis $\operatorname{set}^{34-36}$ (optimized H-O-H angle of $104.12^{\circ}$ and O-H distance of $0.969 \AA$, in good agreement with experimental values $\mathrm{H}-\mathrm{O}-\mathrm{H}$ angle of $104.48^{\circ}$ and $\mathrm{O}-\mathrm{H}$ distance of $0.958 \AA$ ). The basis set used in the calculations is an aug-cc-pcVQZ Gaussian basis set corresponding to a primitive $(16 s, 10 p, 6 d, 4 f, 2 g)$ set contracted to $[9 s, 8 p, 6 d, 4 f, 2 g] .{ }^{37}$ In Table 1 , the computed non-relativistic BEs are reported at the HF level of calculation and applying three different DFT methods. Those include a GGA derivation of the exchange-correlation functional, the PBE functional, ${ }^{33}$ and two hybrid implementations, the 3-parameter Becke-Lee-Yang-Parr (B3LYP) ${ }^{38,39}$ and the hybrid PBE (PBE0), ${ }^{40}$ with inclusion of $20 \%$ and $25 \%$ of HF exchange, respectively. From Table 1 it can be seen that BEs derived from the application of Koopman's theorem for $\varepsilon_{\mathrm{i}}-\mathrm{KS}$ are meaningless and do not represent initial state effects. Instead, these effects can be accounted for by computing the $\mathrm{BE}$ by means of a $\mathrm{FO}$ wavefunction, where the core hole is frozen as stated in Eq. 5. In such a case, initial state effects computed by DFT are similar to the value obtained by HF calculations, where BEs obtained by KT are well-defined and have physical meaning. BEs computed by the $\triangle \mathrm{SCF}$ approach include final state effects and are closer to the experimental value, $539.70 \mathrm{eV} .^{41}$ As a matter of fact, final state effects can be evaluated by calculating the relaxation energy as the difference between the $\triangle \mathrm{SCF} \mathrm{BE}$ and the initial state BE computed as $\mathrm{BE}(\mathrm{FO})$. Relaxation energy at the $\mathrm{HF}$ level is $20.48 \mathrm{eV}$ while this magnitude is $20.70 \mathrm{eV}$ for PBE calculations. Hybrid functionals give slightly lower values, 20.61 and 20.64 for B3LYP and PBE0, respectively. As shown in Table 1, final state $\triangle$ SCF BEs computed by HF and B3LYP show rather better agreement with experiment that PBE and PBE0 results. However, it has to be noticed that relativistic effects are not accounted for. As commented previously, relativistic corrections to the BE can be accurately estimated by fully relativistic atomic calculations. For the $\mathrm{O}(1 s)$ core hole of the isolated $\mathrm{O}$ atom, relativistic 
effects increase the BE by $0.45 \mathrm{eV}$. Hence, inclusion of the atomic relativistic corrections in the $\mathrm{O}(1 s) \mathrm{BE}$ of the water molecule brings the HF value to $539.63 \mathrm{eV}$ and the B3LYP result to $539.84 \mathrm{eV}$, both very close to the $539.70 \mathrm{eV}$ measured value.

Calculations based on the GW quasiparticle approach have also been carried out using the same geometry as in the HF and DFT calculations but results have been extrapolated to the complete basis set limit. Different $\mathrm{GW}$ approaches have been considered: $\mathrm{G}_{0} \mathrm{~W}_{0}$ with linearized quasiparticle equation, starting either with PBE or PBE0 densities, and quasiparticle self-consistent GW. The $\mathrm{O}(1 s)$ core hole BE corresponding to the linearized $\mathrm{G}_{0} \mathrm{~W}_{0}$ method starting with PBE and PBE0 densities are respectively, 529.45 and $534.90 \mathrm{eV}$, whereas the self-consistent implementation improves the value to $542.13 \mathrm{eV}$ providing a rather accurate result yet $2.4 \mathrm{eV}$ larger than the experimental one.

\section{A survey of examples and perspectives}

In this section we critically revise the so far accumulated literature on core level BEs prediction by the above-commented methodologies, with a final overview on future work and applicability on materials and surface sciences. As aforementioned, HF method has been and is the working horse in obtaining core level BEs, either within the FO approximation to evaluate initial state effects, or by $\triangle \mathrm{SCF}$ methodology to acquire final state BEs. The HartreeFock orbital energies $\left(\mathrm{KT}-\varepsilon_{\mathrm{i}}\right)$ and $\triangle \mathrm{SCF}$ values have been used to understand XPS of a broad range of systems, although here we will focus on several systematic studies evaluating the peculiarities and suitability of HF to predict core level BEs. One of these earliest systematic works is that of Besley et al., ${ }^{42}$ who studied the performance of HF for 1s core level BEs of a series of 14 simple organic molecules containing $\mathrm{C} \rightarrow \mathrm{F}$ elements using a 6-311G** basis set. Results show a mean absolute deviation (MAD) with respect to experiments of $0.60 \mathrm{eV}$, a value which is only slightly changed $(0.64 \mathrm{eV})$ when using a larger, more complete, ccpCVQZ basis set. This indicates that basis set convergence seems to be reached. However, 
better values are obtained when using a fully uncontracted basis set with the same primitive set (named u6-311G**) which yielded a somewhat better performance with a MAD of 0.44 $\mathrm{eV}$. This is not surprising since an uncontracted basis allows for a better relaxation of the core electron density upon ionization

A posterior study, for a series of $19 \mathrm{~N}(1 s)$ core levels of $17 \mathrm{~N}$-containing organic molecules, ${ }^{16,17}$ evaluated the performance of the HF $\triangle \mathrm{SCF}$ methodology using a fully uncontracted basis set of polarized valence triple zeta (pVTZ) quality for the $\mathrm{N}$ atom. Results consistently yielded a MAD value of $0.8 \mathrm{eV}$, highlighting how the choice of the explored data set, even the particular core level can lead to different accuracies. Indeed, further work on the same spirit expanded the study to $\mathrm{C} \rightarrow \mathrm{F}$ elements -20 molecules and 39 core levels - , with a slightly improved MAD of $0.67 \mathrm{eV} .^{43}$ In that work it was highlighted that the agreement between theory and experiment is high for $\mathrm{C}(1 s)$, although it gets slightly worse for the other elements. In the most exhaustive study published to date in this subject, a total of 68 molecules containing elements from B to F were explored with a total of $1891 \mathrm{~s}$ core level BEs analyzed. ${ }^{21}$ At the HF level of theory, results yielded MAD values of $0.22,0.30,0.49$, 0.79 , and $1.09 \mathrm{eV}$ for $\mathrm{B} \rightarrow \mathrm{F}$, respectively, with an overall performance with a MAD value of $0.44 \mathrm{eV}$.

Notice that such discrepancies with experimentally measured BEs are explained, in part, due to the absence of relativistic effects in the calculations, which can actually be significant for such low-energy core levels and become more important as $\mathrm{Z}$ increases. Inclusion of relativistic effects bridges the disagreement gap by $0.06,0.13,0.25,0.45$, and $0.75 \mathrm{eV}$ for $\mathrm{B} \rightarrow \mathrm{F}$, respectively, and so, when accounted for reduces the MAD to $0.3 \mathrm{eV}$ only. Notice that these explicit relativistic corrections differ from those obtained from previously raw approximations where the correction, $C_{r e l}{ }^{44}$ is evaluated as

$$
C_{r e l}=K I_{n r}{ }^{N}
$$


where $K=2.198 \cdot 10^{-7}, N=2.178$, and $I_{n r}$ the non-relativistic BE with the final value of $C_{r e l}$ in $\mathrm{eV}$. By this approximation, the relativistic corrections are about $0.02,0.05,0.10,0.19$, and $0.34 \mathrm{eV}$ for $1 \mathrm{~s}$ cores in atoms $\mathrm{B} \rightarrow \mathrm{F}$. These are clearly underestimated by more than half with respect to the fully relativistic Dirac-Fock calculations, a somehow expected issued when using a phenomenological equation. ${ }^{44}$ To a large extent, relativistic and electron correlation effects not included in the HF wavefunction cancel out when considering $\triangle \mathrm{BEs}$. Along this line, the performance on HF $\triangle B E s$ on a reduced set of data yield MAD values of $0.38 \mathrm{eV}$, in comparison to the value of $0.67 \mathrm{eV}$ for absolute core level BEs. ${ }^{43}$ By considering a more complete set of data, the $\triangle \mathrm{BEs}$ MAD drop to values of $0.27 \mathrm{eV}$, and, as expected, no significant discrepancies are found for the different considered elements.

Despite HF has been a working horse in evaluation of core level BEs, other methods have been explored as well. It is worth to mention that Besley et $a l^{42}$ also explored the performance of second order Møller-Pleset (MP2), which yield slightly better results than HF, with a MAD with $\mathrm{u} 6-311 \mathrm{G}^{* *}$ of $0.36 \mathrm{eV}$. However, the largest part of the exploratory studies aimed at using DFT for the calculation of core level BEs. This is because, in principle, DFT based methods include electron correlation effects lacking in HF. In that sense, the original article by Besley et $a l^{42}$ showed that B3LYP exchange-correlation (xc) functional ${ }^{38,39}$ yielded $^{4}$ MAD values of solely $0.25 \mathrm{eV}$ using the $\mathrm{u} 6-311 \mathrm{G}^{* *}$ basis set. A posterior study using the hybrid B3LYP density functional on the $\mathrm{N} 1 s$ data set, ${ }^{16}$ came out with similar conclusions and, in line with arguments in the previous section, also clearly demonstrated that it is not correct to interpret $\mathrm{KS}-\varepsilon_{\mathrm{i}}$ as estimate of initial state core level BEs. ${ }^{17}$

The literature contains a number of works evaluating the performance of a wide variety of xc density functionals in estimating BEs from several molecular data sets, mostly focusing on $1 s$ levels of main group elements. Cavigliasso and Chong ${ }^{45}$ evaluated the 
performance of 10 possible combinations of exchange and correlation parts within the local density approximation (LDA) or the GGA approximation on a set of $171 s$ core levels of 13 molecules, where different basis sets, encompassing cc-pVnZ type up to quintuple zeta (ccpV5Z) and scaled basis sets of pVTZ type yielded converged basis set limit results of $0.15 \mathrm{eV}$ for the combination of Perdew-Wang 1986 exchange (PW86) ${ }^{46}$ with Perdew-Wang 1991 correlation (PW91). ${ }^{47}$ A posterior study by Takahata and Chong ${ }^{48}$ further confirmed this, by evaluating $591 s$ core level BEs of 22 main group molecules, contemplating 35 different combinations of exchange and correlation functionals within LDA and GGA with increasing basis sets up to QZ4P level, and including the aforementioned $C_{r e l}$ correction. The best performance was found again for the PW86/PW91 combination, with a MAD value of solely $0.16 \mathrm{eV}$. Other authors, by evaluating $27 \mathrm{xc}$ combinations on $271 \mathrm{~s}$ core levels of 18 molecules suggested as well the PW86/PW91 combination in a 60:40 ratio, with a MAD value of $0.20 \mathrm{eV} .^{49}$

Indeed, further work on this field targeted the description of other type of core levels. In particular, we mention the extensive work of Segala et al. ${ }^{50}$ in describing $2 p$ core levels for 145 cases of Si, P, S, Cl, and Ar containing molecules. These authors evaluated the core level BEs at the $\triangle \mathrm{SCF}$ approach by considering $26 \mathrm{xc}$ functionals and including relativistic effects through the Zeroth Order Regular Approximation (ZORA). ${ }^{51,52}$ At variance with $1 s$ BEs, the best performance is found for a combination of OPTX functional for exchange, ${ }^{53}$ with LeeYang-Parr (LYP) correlation, ${ }^{39}$ with a MAD value of $0.26 \mathrm{eV}$, whereas for this type of orbitals the performance of PW86/PW91 was poorer with a MAD value of $0.45 \mathrm{eV}$. Minnesota functionals were evaluated by Pueyo Bellafont et al. ${ }^{43}$ finding that hybrid M11 ${ }^{54}$ and metaGGA M06-L ${ }^{55}$ excelled over other with MAD values of $0.33 \mathrm{eV}$, thus, better to HF or B3LYP in the same study. The same MAD value of $0.33 \mathrm{eV}$ was found for the metaGGA xc of Tao-Perdew-Staroverov-Scuderia (TPSS), ${ }^{56} 0.21 \mathrm{eV}$ when including relativistic effects. 
From the discussion so far it is clear that HF and the different DFT based methods exhibit a similar performance, at least as far as this is measured from the calculated MADs ranging from 0.2 to $0.8 \mathrm{eV}$ depending on the method and on the ionized core. In relative terms the error is very small since these BEs are of the order $300-600 \mathrm{eV}$ but in absolute terms this may still be too large when aiming at interpreting XPS data where the limit of accuracy of High-Resolution Photoemission Electron Spectroscopy (HR-PES) is of $\sim 0.1 \mathrm{eV}$. Regarding $\triangle \mathrm{BE}$ values, DFT based methods seem to surpass in efficiency $\mathrm{HF},{ }^{43}$ with MAD values as small as $0.16 \mathrm{eV}$ for hybrid functionals B3LYP and M06, ${ }^{57}$ whereas other metaGGA functionals such as M06-L and M11-L ${ }^{58}$ yield MAD values of $0.18 \mathrm{eV}$, and TPSS displays a value of $0.25 \mathrm{eV}$ on the 185 systems data set, ${ }^{21}$ compared to a value of $0.24 \mathrm{eV}$ for the GGA of Perdew-Burke-Ernzerhof (PBE), ${ }^{33}$ and a value for HF of $0.27 \mathrm{eV}$.

At this point, the performance of the $\triangle \mathrm{SCF}$ approach, either using HF or DFT based total energy calculations, in predicting BEs and $\triangle \mathrm{BEs}$ has been clearly established indicating that quite accurate predictions for small to medium size organic molecules is to date at hand. These theoretical approaches are equally applicable to more complex systems involving transition metals, lanthanides or actinides although the calculation of core level binding energies is likely to be complicated by the presence of multiplet splitting and/or intrinsic satellites; the interested reader is addressed to the review papers by Bagus et al. ${ }^{13,15}$

Further steps are necessary where aiming at predicting these values for such molecules interacting with solid surfaces. This is especially the case when crystalline solids are described using codes exploiting periodic boundary conditions. As already commented, the ionization of one core electron in a given atom leads to a charged periodic cell, with the concomitant problematic convergence issues along with possible artefacts in the calculated values. In fact, a common way out of the problem consists in creating a countercharge inside the cell which can lead to unrealistic core level BEs and $\triangle \mathrm{BEs}$ as a result of the perturbation 
created by the artificially added electric field. An additional, not minor, problem is that most of the available and broadly used periodic codes use pseudopotentials to take into account the effect of the atomic core in the valence electron density. In computational materials science, the Projector Augmented Wave (PAW) ${ }^{59}$ method is one of the most commonly used.

Despite this limitation, some steps towards approximate $\triangle \mathrm{SCF}$ calculation in periodic calculation have been reported in the literature. In particular we highlight the paramount work of Pehlke and Scheffler ${ }^{60}$ on the use of a pseudopotential approach to study of core holes at the $\mathrm{Si}(001)$ and $\mathrm{Ge}(001)$ surfaces and the more recent work of Ljungberg et al. ${ }^{18}$ within the GPAW. These authors point the necessity in carrying out spin-polarized calculations when exciting one hole electron, otherwise variations of up to $10 \mathrm{eV}$ can be found, as reported for the $\mathrm{H}_{2} \mathrm{O}$ molecule. This is quite a surprising result since spin restricted (open shell) and unrestricted calculations indicate that the effect of spin polarization is in this case negligible. In spite of this, their implementation yielded a $\mathrm{BE}$ estimation only $0.8 \mathrm{eV}$ far from the experimental value. An alternative implementation has been proposed by Köhler and Kresse ${ }^{61}$ although actually models X-ray Absorption Spectroscopy (XAS) rather than XPS, given that the excited electron is placed in the molecular Lowest Unoccupied Molecular Orbital (LUMO), or, in periodic systems, in the band right above the Fermi level. The only systematic study on this procedure considered the $1851 s$ data set and focused in initial and finals state effects as predicted from the approach of Köhler and Kresse, ${ }^{61}$ as well as the Janak-Slater (JS) approximation, in which only half an electron is excited. The authors also inspected the case of removing the electron - half the electron in JS - from the system, with the charge in the unit cell counteracted by a uniform background of opposite charge. Results revealed that fully removal of one electron lead to better agreement with experiment for $\triangle \mathrm{BEs}$ although this approach does not allow to obtain absolute values for the core level BEs. Applying the JS approach while removing the excited half electron lead to MAD values of 0.37 and $0.21 \mathrm{eV}$ 
for the $\triangle \mathrm{BEs}$ with PBE and TPSS xc functionals, respectively, ${ }^{62}$ indicating that, as long as $\triangle \mathrm{BEs}$ are concerned, this is a sound approach.

In spite of the potential of these PAW based approach to estimate $\triangle \mathrm{BEs}$, applications to surface science are scarce with pioneering studies on $\mathrm{CO}$ adsorbed on $\mathrm{Ni}(001)^{60}$ or $\mathrm{Rh}(111)$ surfaces. ${ }^{61}$ Subsequent successful applications involve interpreting HR-XPS of $\mathrm{SO}_{\mathrm{x}}$ species on Pd surfaces, ${ }^{63,64}$ or the different attachment modes of graphene on Ni(111). ${ }^{65}$ However, the most spread approximation found in the literature to evaluate BEs and $\triangle \mathrm{BEs}$ on solid systems or molecules interacting with crystalline surfaces involves $\triangle \mathrm{SCF}$ calculations (either at the HF or DFT levels) using cluster models. ${ }^{13}$ Along this line an extended set of examples are found in the literature, from the evaluation of the interaction of halides on $\mathrm{Pt}(111)$, using effective core potentials in the surrounding metal atoms not in direct contact to the halide atom to reduce the computational burden, ${ }^{66}$ passing by the effect on $\mathrm{O}(1 s)$ in different alkaline and alkaline-earth oxides, ${ }^{67}$ to the effect of strain in the core levels BEs of Pt-Ru nanoparticles. ${ }^{68}$ Indeed, coupling sufficiently accurate estimates of BEs and $\triangle B E s$ to XPS, PES, and HR-XPS experiments is of great importance, given that such experiments are broadly in use in solid state physics, materials science, nanotechnology, and catalytically relevant systems. ${ }^{69}$ However, the theoretical methods described in the present perspective have to be further tested on an extended variety of systems, including proper modelling, to ascertain the degree of applicability and reliability, a point that definitely is a matter of future research.

\section{Conclusions}

Core level Binding Energies (BE) are available from X-ray Photoemission Spectroscopy (XPS) and provide important information regarding the chemical environment of the atoms is particular systems. Yet, direct interpretation of XPS is not always possible, especially when different and even contradictory hypothesis can be formulated. In these cases, 
theory is like to provide the clue to decide among the different interpretations. However, theoretical values need to be accurate enough and this has triggered a considerable amount of work that has been critically assessed in the present perspective article.

The state-of-the- art methods of computational chemistry allow for accurate predictions of core level BEs of $1 s$ cores of main group element containing molecular systems. The mean absolute error for Delta Self-Consistent Field $(\triangle \mathrm{SCF})$ calculations at the Hartree-Fock level is roughly of $0.5 \mathrm{eV}$. This may be larger or smaller for $\Delta \mathrm{SCF}$ calculations using Density Functional Theory (DFT) based methods with average error of the order of 0.8 $\mathrm{eV}$ for the Perdew-Burke-Ernzerhof Generalized Gradient Approximation (GGA) type functional or slightly larger than $0.2 \mathrm{eV}$ for the Tao-Perdew-Staroverov-Scuseria meta-GGA one. ${ }^{17,21}$ Errors on core level binding energy shifts being generally smaller due to error cancellation effects, most specifically of relativistic effects, which are quite specific of the atomic core.

The interpretation of orbital energies as an approximation to initial state core level binding energy holds for Hartree-Fock calculations but it is not correct for DFT based ones although a proper definition of initial state effects is also possible. ${ }^{17}$ On the other hand, orbital energies, either at Hartree-Fock or DFT levels, provide a rather good estimate of core level binding energy shifts. ${ }^{16}$ This provides rather fast and reliable information when handling extended systems for which $\triangle \mathrm{SCF}$ calculations may be cumbersome. Methods to take into account final state effects on the core level shift in periodic systems have also been proposed and briefly discussed showing, however, the difficulty to obtain absolute values of the core level binding energies. In this respect, the emerging $G W$ techniques are likely to provide a reliable and physically meaningful result even if at a quite high computational cost. 


\section{Acknowledgements}

This research was supported by the Spanish MINECO/FEDER CTQ2015-64618-R grant and, in part, by Generalitat de Catalunya (grants 2014SGR97 and XRQTC) and by the NOMAD Center of Excellence project, which received funding from the European Union's Horizon 2020 research and innovation programme under grant agreement No 676580. F.V. thanks MINECO for a postdoctoral Ramón y Cajal (RyC) research contract (RYC-2012-10129) and F.I. acknowledges additional support from the 2015 ICREA Academia Award for Excellence in University Research. 
Scheme 1. Processes of $1 \mathrm{~s}$ core level photoemission upon X-ray hv radiation on $\mathrm{H}_{2} \mathrm{O}$ molecule (upper part) and on a materials crystal surface (lower part). In the case of the surface, the excited electron places first in the Fermi level, $\mathrm{E}_{\mathrm{F}}$, upon which is emitted to vacuum surpassing the energy cost of the surface work function, $\phi$.

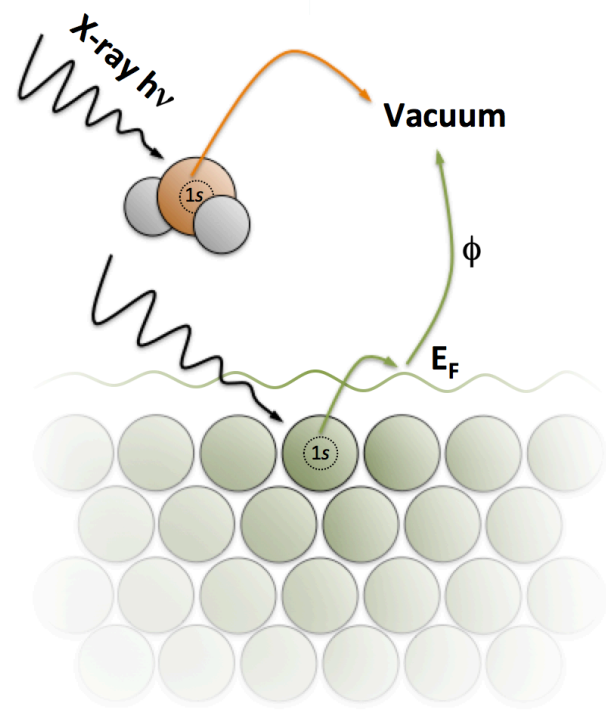


Table 1. $\mathrm{O}(1 s)$ core level binding energy for the water molecule computed applying Koopman's theorem (KT), considering a frozen core hole as obtained from Eq. 5, BE(FO), and calculated as the energy difference between the final and initial states (Eq. 3), $\mathrm{BE}(\triangle \mathrm{SCF})$. All values are in $\mathrm{eV}$. The experimental value is $539.70 \mathrm{eV}$.

\begin{tabular}{lccc}
\hline & KT $\left(-\varepsilon_{\mathrm{i}}\right)$ & BE(FO) & BE $(\Delta$ SCF $)$ \\
\hline HF & 559.65 & 559.65 & 539.18 \\
PBE & 510.42 & 559.34 & 538.64 \\
PBE0 & 522.79 & 559.54 & 538.91 \\
B3LYP & 520.68 & 560.00 & 539.39 \\
\hline
\end{tabular}




\section{References}

1 C. Nordling, E. Sokolowski, K. Siegbahn, Phys. Rev., 1957, 105, 1676.

2 K. Siegbahn, C. Nordling, A. Falhman, R. Nordberg, K. Hamrin, J. Hedman, G. Johansson, T. Bergmark, S. E. Karlsson, I. Lindgren, B. Lindberg, ESCA-Atomic, Molecular and Solid State Structure Studied by Means of Electron Spectroscopy, Alwmvist and Wiksells Boktryckeri AB, Uppsala, Sweden, 1967.

3 K. Siegbahn, C. Nordling, G. Johansson, J. Hedman, P. F. Hedén, K. Hamrin, U. Gelius, T. Bergmark, L. O. Werme, R. Manne, Y. Baer. ESCA-Applied to Free Molecules, North-Holland, Amsterdam, 1969.

4 E. Sokolowski, C. Nordling, K. Siegbahn, Phys. Rev., 1958, 110, 776.

5 P. S. Bagus, F. Illas, G. Pacchioni, F. Parmigiani, J. Electr. Spectrosc. Relat. Phenom., 1999, 100, 215.

6 F. A. Delesma, M. Van den Bossche, H. Grönbeck, P. Calaminici, A. M. Köster, L. G.M. Pettersson, ChemPhysChem, 2017, doi: 10.1002/cphc.201701135.

7 Y. Lykhach, T. Staudt, M. P. A. Lorenz, R. Streber, A. Bayer, H. P. Steinrück, J. Libuda, ChemPhysChem, 2010, 11, 1496.

8 M. Happel, N. Luckas, F. Viñes, M. Sobota, M. Laurin, A. Görling, J. Libuda, J. Phys. Chem. C, 2011, 115, 479.

9 I. Niedermaier, C. Kolbeck, N. Taccardi, P. S. Schulz, J. Li, T. Drewello, P. Wasserscheid, H. P. Steinrück, F. Maier, ChemPhysChem, 2012, 13, 1725.

10 P. S. Bagus, Phys. Rev., 1965, 139, A619.

11 A. A. Bakke, H. W. Chen, W. L. Jolly, J. Electron. Spectrosc. \& Rel. Phenom., 1980, 20, 333.

12 J. Casanovas, J. M. Ricart, J. Rubio, F. Illas, J. M. Jimenez Mateos, J. Am. Chem. Soc., 1996, 118, 8071 .

13 P. S. Bagus, E. S. Ilton, C. J. Nelin, Surf. Sci. Rep., 2013, 68, 273.

14 B. Hammer, J. K. Nørskov, Adv. Catal., 2000, 45, 71

15 P. S. Bagus, C. R. Brundle, F. Illas, F. Parmigiani, G. Polzonetti, Phys. Rev. B, 1991, 44, 9025 .

16 N. Pueyo Bellafont, F. Illas, P. S. Bagus, Phys. Chem. Chem. Phys., 2015, 17, 4015.

17 N. Pueyo Bellafont, P. S. Bagus, F. Illas, J. Chem. Phys., 2015, 142, 214102.

18 M. P. Ljungberg, J. J. Mortensen, L. G. M. Petterson, J. Electron. Spectrosc. Relat. Phenom., 2011, 184, 427. 
19 M. Segala, D. P. Chong, J. Electron Spectrosc. Relat. Phenom., 2009, 171, 18.

20 Y. Takahata, D. P. Chong, J. Electron Spectrosc. Relat. Phenom. 2003, 133, 69.

21 N. Pueyo Bellafont, F. Viñes, F. Illas, J. Chem. Theo. Comput., 2016, 12, 324.

22 P. Duffy, D.P. Chong, M. E. Casida, D. R. Salahub, Phys. Rev. A 1994, 50, 4707.

23 O. V. Gritsenko, E. J. Baerends, J. Chem. Phys. 2002, 117, 9154.

24 J. V. Ortiz, Int. J. Quantum Chem. 2004, 100, 1131.

25 Y. Öhrn, J. V. Ortiz, Mol. Phys. 2010, 108, 2871 and references therein

26 L. Hedin, Phys. Rev. 1965, 139, A796.

27 F. Aryasetiawan, O. Gunnarsson, Rep. Prog. Phys., 1998, 61, 237.

28 S. Faleev, M. van Schilfgaarde, T. Kotani, Phys. Rev. Lett., 2004, 93, 126406.

29 M. J. van Setten, F. Caruso, S. Sharifzadeh, X. Ren, M. Scheffler, F. Liu, J. Lischner, L. Lin, J. R. Deslippe, S. G. Louie, C. Yang, F. Weigend, J. B. Neaton, F. Evers, P. Rinke, J. Chem. Theory Comput., 2015, 11, 5665.

30 M. J. van Setten, R. Costa, F. Viñes, F. Illas, J. Chem. Theory and Comput., 2018, 14, 877 ,

31 L. Hedin, A. Johansson, J. Phys. B, 1969, 2, 1336.

32 N. Pueyo Bellafont, P. S. Bagus, C. Sousa, F. Illas, J. Chem. Phys., 2017, 147, 024106.

33 J. P. Perdew, K. Burke, M. Ernzerhof, Phys. Rev. Lett., 1996, 77, 3865.

34 V. Blum, R. Gehrke, F. Hanke, P. Havu, V. Havu, X. Ren, K. Reuter, M. Scheffler, Comput. Phys. Commun., 2009, 180, 2175.

35 V. Havu, V. Blum, P. Havu, S. Matthias, J. Comput. Phys., 2009, 228, 8367.

36 X. Ren, P. Rinke, V. Blum, J. Wieferink, A. Tkatchenko, A. Sanfilippo, K. Reuter, M. Scheffler, New J. Phys., 2012, 14, 053020.

37 R. A. Kendall, T. H. Dunning Jr., R. J. Harrison, J. Chem. Phys., 1992, 96, 6796.

38 A. D. Becke, J. Chem. Phys., 1993, 98, 5648.

39 C. Lee, W. Yang, R. G. Parr, Phys. Rev. B: Condens. Matter Mater. Phys., 1988, 37, 785.

40 C. Adamo, V. Barone, J. Chem. Phys., 1999, 110, 6158.

41 A. A. Bakke, H. W. Chen, W. L. Jolly, J. Electron Spectrosc. Relat. Phenom., 1980, 20, 333.

42 N. A. Besley, A. T. B. Gilbert, P. M. W. Gill, J. Chem. Phys., 2009, 130, 124308.

43 N. Pueyo Bellafont, G. Álvarez Saiz, F. Viñes, F. Illas, Theor. Chem. Acc., 2016, 135, 35.

44 D. P. Chong, J. Chem. Phys., 1995, 103, 1842.

45 G. Cavigliasso, D. P. Chong, J. Chem. Phys., 1999, 111, 9485. 
46 J. P. Perdew, Y. Wang, Phys. Rev. B, 1986, 33, 8800.

47 J. P. Perdew, Y. Wang, Phys. Rev. B, 1992, 46, 12947.

48 Y. Takahata, D. P. Chong, J. Electr. Spectrosc. Relat. Phenom., 2003, 133, 69.

49 O. Takahashi, L. G. M. Pettersson, J. Chem. Phys., 2004, 121, 10339.

50 M. Segala, Y. Takahata, D. P. Chong, J. Electron. Spectrosc. Relat. Phenom., 2006, 151, 9.

51 Ch. Chang, M. Pelissier, Ph. Durand, Phys. Scr., 1986, 34, 394.

52 E. van Lenthe, E. J. Baerends, J. G. Snijders, J. Chem. Phys., 1993, 99, 4597.

53 N. C. Handy, A. J. Cohen, Mol. Phys., 2001, 99, 403.

54 R. Peverati, D. G. Truhlar, J. Phys. Chem. Lett., 2011, 2, 2810.

55 Y. Zhao, D. G. Truhlar, J. Chem. Phys., 2006, 125, 194101.

56 J. Tao, J. P. Perdew, V. N. Staroverov, G. E. Scuseria, Phys. Rev. Lett., 2003, 91, 146401.

57 Y. Zhao, D. G. Truhlar, Theor. Chem. Acc., 2006, 120, 215.

58 R. Peverati, D. G. Truhlar, J. Phys. Chem. Lett., 2012, 3, 117.

59 P. E. Blöchl, Phys. Rev. B, 1994, 50, 17953.

60 E. Pehlke, M. Scheffler, Phys. Rev. Lett., 1993, 71, 2338.

61 L. Köhler, G. Kresse, Phys. Rev. B, 2004, 70, 165405.

62 N. Pueyo Bellafont, F. Viñes, W. Hieringer, F. Illas, J. Comput. Chem., 2017, 38, 518.

63 K. Gotterbarm, N. Luckas, O. Höfert, M. P. A. Lorenz, R. Streber, C. Papp, F. Viñes, H.P. Steinrück, A. Görling, J. Chem. Phys., 2012, 136, 094702.

64 N. Luckas, K. Gotterbarm, R. Streber, M. P. A. Lorenz, O. Höfert, F. Viñes, C. Papp, A. Görling, H.-P. Steinrück, Phys. Chem. Chem. Phys., 2011, 13, 16227.

65 W. Zhao, S. M. Kozlov, O. Höfert, K. Gotterbarm, M. P. A. Lorenz, F. Viñes, C. Papp, A. Görling, H.-P. Steinrück, J. Phys. Chem. Lett., 2011, 2, 759.

66 P. S. Bagus, C. Wöll, A. Wieckowski, Surf. Sci., 2009, 603, 273.

67 C. Di Valentin, R. Ferullo, R. Binda, G. Pacchioni, Surf. Sci., 2006, 600, 1147.

68 A. Lewera, W. P. Zhou, R. Hunger, W. Jaegermann, A. Wieckowski, S. Yockel, P. S. Bagus, Chem. Phys. Lett., 2007, 447, 39.

69 C. Papp, H.-P. Steinrück, Surf. Sci. Rep., 2013, 68, 446. 